\title{
Research on Stage Visual-Effect Equipment Control System based on Android
}

\author{
Yujian Jiang ${ }^{1,2,3}$, Ben Cheng ${ }^{1,2,3}$, Wei Jiang ${ }^{1,2,3}$,Shaobo Wang ${ }^{1,2,3}$ \\ ${ }^{1}$ Information Engineering School, Communication University of China \\ ${ }^{2}$ Key Laboratory of Acoustic Visual Technology and Intelligent Control System, Ministry of Culture \\ ${ }^{3}$ Beijing Key Laboratory of Modern Entertainment Technology Beijing, China
}

Keywords: stage visual-effect equipment; android; C/S structure; socket

\begin{abstract}
Aim at a common situation that Control Equipment of Stage Visual-Effect has been heavy, this paper proposes Stage Visual-Effect Equipment Control System consisting of a mobile terminal based on Android. The system is a C/S structure. The system satisfies basic and major functions. An android terminal can debug and control those stage visual-effect equipment, supervise programs. It communicates with a PC through the socket in the wireless network (LAN) cover.
\end{abstract}

\section{Introduction}

With rising aesthetic of the crowed, these designers of stage visual-effect are hoped to give audiences more enjoyment. Stage visual-effect equipment such as fog machines, bubble machines, smoke machines and so on, is playing more and more important roles in varying stage effects and setting off right atmosphere.

When creating a new program for the open stage, it is frequently of the audience to notice the operator when operating those stage visual-effect equipment. And for proscenium, because the stage visual-effect equipment needs covering, operators in the light control room are difficult to find out wrongs in time. Nowadays, most of these stage visual-effect equipment can communicate with control equipment using DMX512 signal through XLR cable. However, the way isn't widespread.

Aim at this problem, this paper proposes a control system of stage Visual-Effect Equipment. Operators can use it to control stage visual-effect equipment when perambulating on the wireless network (LAN) .

\section{Design of System Hardware}

The system has two main functions. 1) debugging and controlling stage effect. 2) supervision of programs. Android is a kind of free and open source code based on the Linux, mainly used in mobile devices. The characteristic of its openness makes its application more and more widely.[1] An android mobile terminal is the main part of the system and a major operation. Operators can drag the sliders to change the DMX512 data and use touch-screens terminals to receive and display the details of the CUE schedule. The mobile terminal is a remote monitor, and the other computer terminal can be used as a local monitor communicated with the lighting console. Android mobile terminals support SD card so to store a large amount of data, CPU multi-thread process data and reduce delays caused by the computation. The friendly UI(user interface) can give operators richer detailed tips. At the same time, touch-screen makes operation more intuitive. Most of these android mobile terminals support WIFI, which can satisfy the requirements of the system for portability and mobility. IEEE $802.11 \mathrm{~b}$ WLAN band of working in 2.4GHz (public spectrum) [2]. Data transfer rates can automatically switch in rates of $11 \mathrm{Mbps}, 5.5 \mathrm{Mbps}, 2 \mathrm{Mbps}, 1 \mathrm{Mbps}$ according to the signal strength or interference.

A computer is an auxiliary hardware of system. On the one hand, a computer can receive and transform data onto a wireless signal. Because most of the special equipment can't accept the wireless signal, only through XLR cable those can accept DMX512 line signal. So the system chooses a computer to accept the wireless signal and transform data. Then through the USB-DMX 
device does the computer connect to the equipment. Stage visual-effect equipment is connecting by a parallel way("hand in hand"). On the other hand, a computer is local supervision terminals.

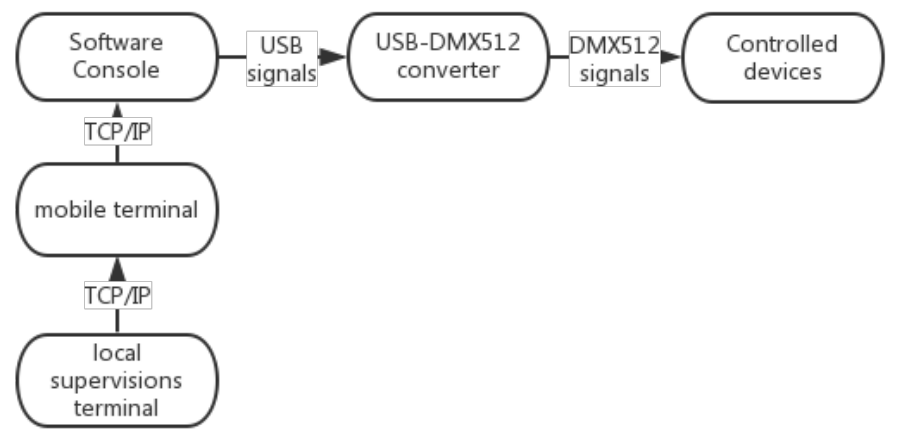

Figure1. Architecture diagram of the system

The system is a C/S structure actually. The system uses TCP/IP to transform data. TCP is a reliable data stream service, using "must confirm with retransmission" technology to realize the reliability of the transmission.[3] A TCP service is really a kind of client/server structure, a service process reads information and response, the client reads response and reports to the user. An android terminal is the client. A computer is a server to communicate with stage special effect equipment through XLR cable. The computer can receive data and converted data to the form conformed to the DMX512 protocol.

\section{Design of System Software}

\section{Overview of the software console.}

The android mobile terminal is the main operation of device and information communication of equipment. The realizations of the function modules are based on Java software. There are mainly four modules: single channel control module, multi-channel control module, cue preview module and real-time monitoring module. The single channel control module uses 512 sliders control channels. Operators can touch the screen to change one or multiple values of channels successively. Multi-channel control module put numerical integration to on a button, and designers select different buttons to choose different materials so as to change all value once. Cue preview module receives Cue list sent by the local supervision terminal, and displays a sheet in the ListView list, updating the information about control on time. Real-time monitoring module receives real-time cue table ID, and displays it on the remote terminal. At the same time the module can count up and display it.

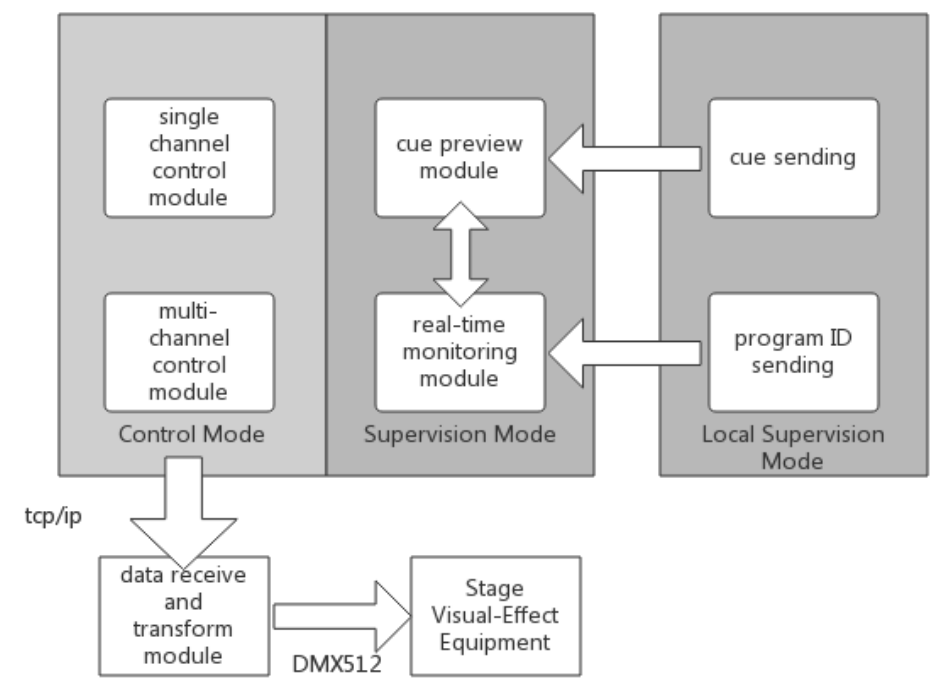

Figure2. Module schematic

Data receive and transform module is realized by computers, and its function is that through LAN does this module receive wireless signal sent by mobile terminals. And then transform into the form following DMX512 signal. 


\section{Workflow of the software console.}

Multi-threading technology reduces response time. UI can be hand in hand with the other work having been active. Currently as no processing tasks, processor time could be given to other tasks. Taking up a lot of processing time of tasks can be periodically given processor time for other tasks.[4] In the system, the establishment of the socket and processor of CUE files also set up by different threads, making programs run smoothly.

The Control Mode comes true the main operation of a mobile terminal. When selecting a single channel module, the terminal changes a channel numerical. When multi-channel module, the current scene shall be integrated into the button so to change 512 channels once. No matter which control modes were selected, the application should establish a socket, then send requests or data. Corresponding the computer terminal establishes a socket and binds port to start listening. After listening to the signals, the data receive and transform module begins to receive data, then transforms data onto the DMX512 signal, lastly closes the socket.

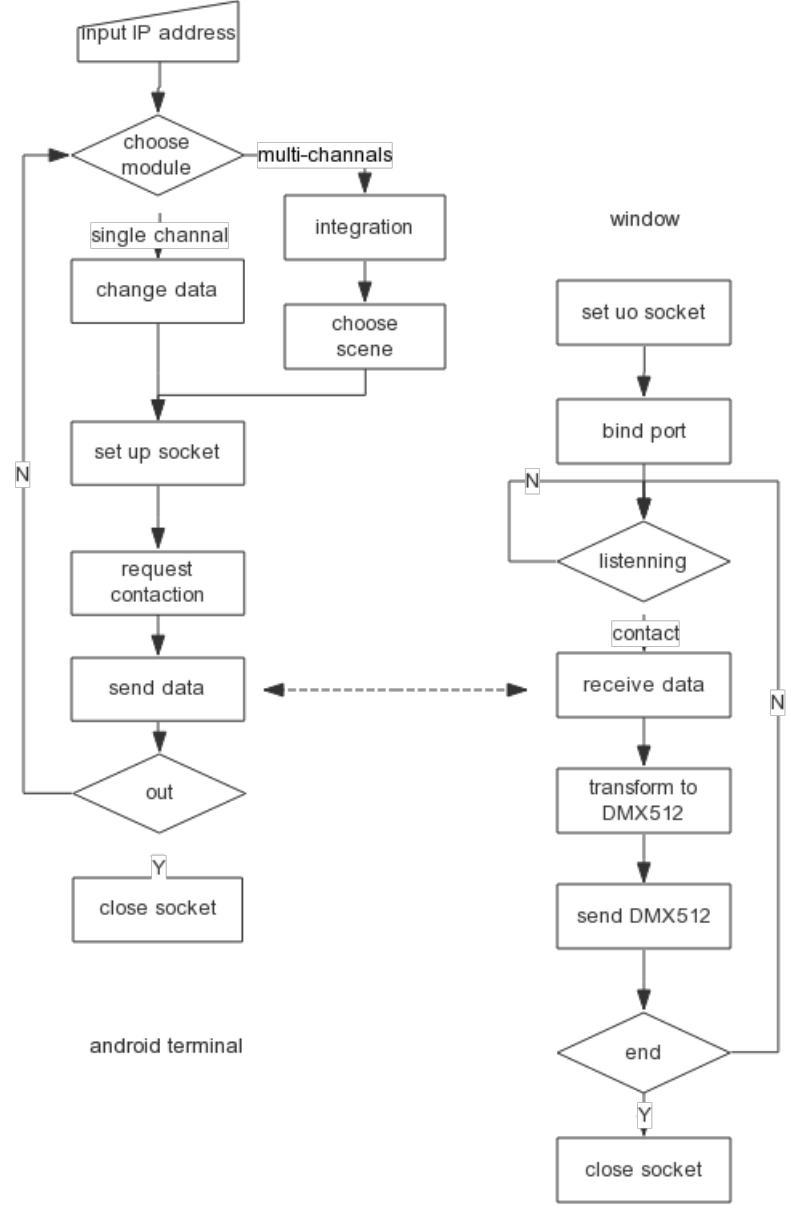

\section{/*pseudocode*/}

Figure3. Workflow of Control Mode

public class SendThread extends Thread \{

public void run()\{

try\{

Socket client = new Socket(IP, 8000);

\}

Writer writer $=$ new OutputStreamWriter(client.getOutputStream()); \}

A mobile is the main device of the Supervision Mode. When choosing a monitor module, the terminal firstly establishes threads of socket and binds port to start listening. When a local supervisions terminal requests connection, the connection establishes successfully. After CUE files having been received, application closes the socket. Then the terminal opens a new processing 
thread to fetch effect objects of a complex text file, and displays it in the list of the mobile, at the same time the terminal closes the thread. Aim for real-time monitoring module, the software sets up a new socket and binds port. When a CUE sheet begins to act, local supervisions terminal requests for connection. After the connection establishes successfully, real-time monitoring module begins to receive the cue sheet real-time IDs sent by local supervisions. At the same time, the remote monitor calls onTimer function, and display the current cue name and count up.

/*pseudocode*/

String tempString = null;

while $(($ tempString $=$ bf.readLine()) $!=$ null $)\{$

switch(count)\{

Case: //scene NO. ||scene name||scene describes||start time

$/ / \mid$ gradient time $\|$ stop time $\}$

\}

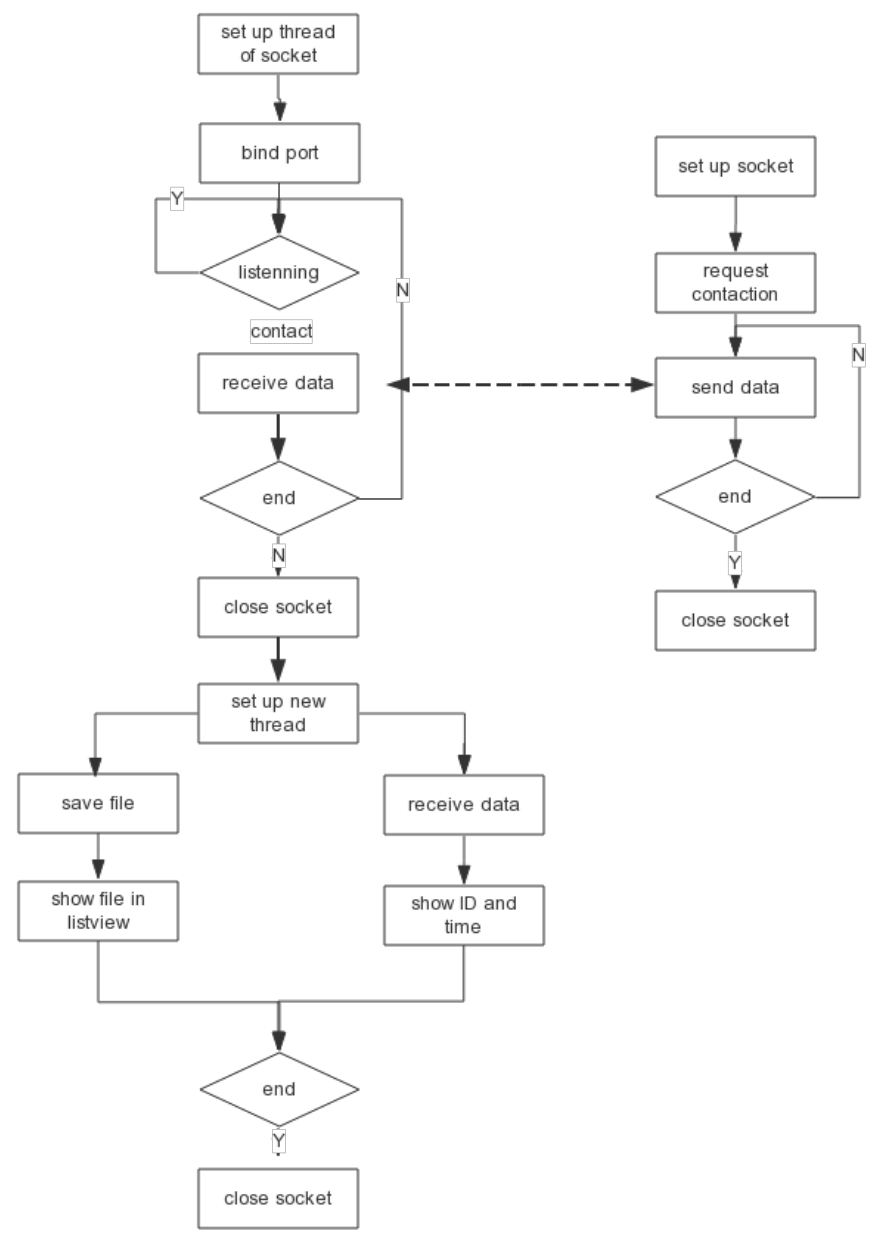

Figure4. Wokflow of Supervision Mode

Dataflow of the software console.

Control Mode was wrote in Java to establish a new class (valueDMX512 class). Single/multiple channel module changes the value and sends it to data receiving module.

/*pseudocode*/

public class valueDMX512 \{

char channal[] = new char [512];

public valueDMX512() \{

//intconfig \}

Data is sent and received through a socket. Data receive and transform module called below function. 


\section{/*pseudocode*/}

SendChannels(WORD ChanCnt, WORD ChanFirst, BYTE *FirstChan); //

SendChannels(512,1,DMX_buf);

Local supervision terminal sends files(text file). Below picture shown in the picture is an example of a program file. This program is made up of multiple scene. Vertical axis is timeline. The actual operation of order is from top to bottom. Format of each cue sheet are "scene NO. \|scene name||scene describes||start time || gradient time\|| stop time || ch1 ||... || ch512".[5]

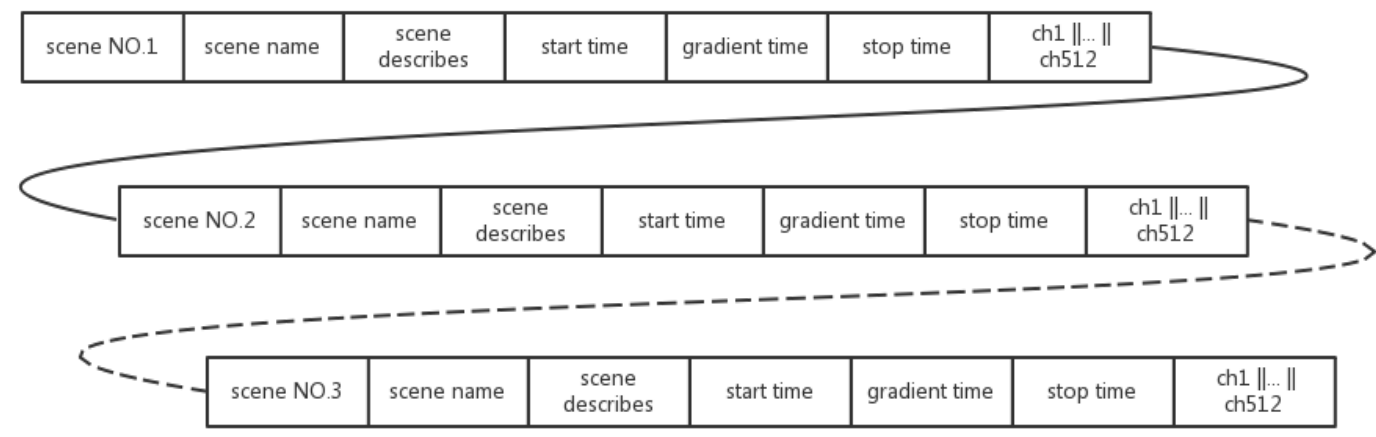

Figure5.Text file of Schematic diagram

Considering that the remote monitoring terminal just needs to check the key message of time and program. So for cue table preview module, "scene NO. ||scene name||scene describes||start time || gradient time|| stop time”, these objects are extracted from the file, creating a new filetrans class.

Then those information are showed in the Listview list.

Table 1. The cue sheet of remote Supervise

\begin{tabular}{|l|c|c|c|c|c|}
\hline NO. & Scene name & scene describes & start time & gradient time & stop time \\
\hline 1 & START & clear & $0 \mathrm{~s}$ & $1 \mathrm{~s}$ & $1 \mathrm{~s}$ \\
\hline 2 & FOG machine & 5 second & $2 \mathrm{~s}$ & $5 \mathrm{~s}$ & $5 \mathrm{~s}$ \\
\hline
\end{tabular}

\section{Conclusion}

Aim of the situation that traditional effects control equipment is difficult to carry, the paper puts forward a system which consists of an android mobile and portable PC linking with stage special effect equipment. Because of wireless network and limited cables, the system can reduce the complex wiring. In the rehearsal stage, it's more flexible and convenient for operators to simultaneously adjust equipment location and debug stage effects. Mobile and computer terminals use wireless to communicate, so the staff can roam in the background of the stage, no longer constrained by the light room, and ensure the timely processing of emergencies avoiding wrongs created by traditional control.

The system improves the experience and pays attention to the human-computer interaction. It keeps the basic function of traditional control equipment. And using mobile on the touch screen as well as the multi-touch simulates mechanical console operation experience.

\section{Acknowledgement}

We would like to thank Beijing Key Laboratory of ModernEntertainment Technology, The 2014 science and technologyinnovation base for Cultivating Development project ( Foundation item: Research on visualization control system for modern entertainment, Project number: Z141101004414045 ) and Engineering Project of CUC (Foundation item:Research on data interaction Vision-Audition stage management system, Project number: 3132015XNG1530) for their help and support. 


\section{References}

[1] Xiong Gang. Based on the design and realization of the android smart phone [D]. Wuhan university of technology, 2010.

[2] Ye Caihong. Based on IEEE802.11 wireless network control system modeling and control [D]. Yanshan university, 2010.

[3] Dong Li. Java technology and its application [M]. Higher education press, 1999

[4] Bo meng Fan XinHua. Java multi-thread applications [J]. Computer knowledge and technology, 2006, $23: 70+96$.

[5] Yujian Jiang,Zhonghao Hu "Research on Data Interaction of stage visual-effect Equipment Control System" Proceedings of 2015 IEEE 5th International Conference on Electronics Information and Emergency Communication, 078-W086(2015) 